\title{
Bitumen paints, an old story with new approach, part-2, water based paints
}

\author{
Mesut Eren ${ }^{1}$, Rıza Aydemir $^{1}$, Hakan Aşkun ${ }^{1}$, Ali Eren Özbey ${ }^{1}$, Murat Orbay ${ }^{1,2}$ \\ ${ }^{1}$ Betek Boya ve Kimya San. A.Ş., İstanbul, Turkey \\ ${ }^{2}$ Istanbul University, Fac. of Engineering (Ret.) İstanbul, Turkey
}

Email address:

mesut.eren@betek.com.tr (M. Eren), hakan.askun@betek.com.tr (H. Aşkun), orbaym@istanbul.edu.tr (M. Orbay)

\section{To cite this article:}

Mesut Eren, Rıza Aydemir, Hakan Aşkun, Ali Eren Özbey, Murat Orbay. Bitumen Paints, an Old Story with New Approach, Part-2, Water Based Paints. Advances in Materials. Vol. 3, No. 3, 2014, pp. 16-21. doi: 10.11648/j.am.20140303.12

\begin{abstract}
Bitumen paints have lost their importance but are still used for large area applications such as tanks and pipelines. It was determined that improving solvent based paint properties by physical or chemical modification by polymers was possible. Applying this approach for water based paints has shown that advance in properties such as hardness and drying times without loss in adhesion and flexibility can be obtained by blending of various polymeric emulsions with bitumen emulsions used for waterproofing membranes. Changes in morphology of the films occurred after blending, indicating interaction between bitumen maltene fractions and polymers. Physical modifications with styrene acrylic emulsions were more rewarding than elastomeric or chemically modifying crosslinking emulsions.
\end{abstract}

Keywords: Bitumen Paints, Water Based, Chemical Modifications, Physical Modifications

\section{Introduction}

$95 \%$ of the bitumen is consumed in road paving, obtained entirely from vacuum distillation residue of petroleum. The rest is employed mainly for preparation of waterproofing membranes and paints. Bitumen is composed of $\mathrm{n}$-heptane soluble maltene (MAL) and insoluble very high molecular weight polycyclic asphaltene (ASP) fractions, responsible for the flexibility and stiffness of the material, respectively. From the chemical point of view, again depending on their solubility in various solvents, MAL can be separated into saturates, aromatics and resins, with increasing molecular weights. Bitumen mainly consists of carbon (80-88 wt.\%) and hydrogen atoms (8-12 wt.\%) In addition, it contains sulphur (0-9 wt.\%) in the form of sulfides, thiols and, to a lesser extent, sulfoxides, nitrogen ( $0-2$ wt.\%) as pyrrolic and pyridinic structures and quinolones and oxygen $(0-2$ wt.\%) in the form of ketones, phenols and carboxylic acids [1].

Recently significant amount of work was devoted to improvement of the asphalt bitumen binder by blending with polymers at elevated temperatures, as well as chemical modifications. They are very well summarized in two excellent reviews by Lesueur [1] and Yildirim [2]. On the other hand, a small volume of bitumen is employed for water proofing of buildings mainly in emulsion form and even smaller one, for solvent based metal coatings. These paints were widely used for iron and steel structures till the second half of last century, but since they show drying time, hardness or flexibility deficiencies, were eventually replaced by paints based on better performing modern polymers. Thus very little research on bitumen paints was carried out recently and the last example was a modification with cashew nut shell liquid-formaldehyde resin, published in 2001 [3].

Bitumen emulsions are oil in water type, and although simple neutralization by a base may be sufficient for emulsification, usually about $1 \%$ cationic or anionic surfactant and bentonite added for stabilization and thickening. They are employed as pavement binder or water proofing membrane preparation.

A literature survey has shown that there is no mention of their employment as binder in water based surface coatings. The trend of the decade, as well as the regulations, promotes the search for transformation to low VOC paints and varnishes. Since previous research on solvent based bitumen paints [4] has shown that modification with polymers, along with proper formulations, can improve bitumen paint properties, preparation of water based bitumen paints by blending commercial bitumen emulsions 
with various polymer emulsions was attempted.

This paper deals with physical, and chemical modifications of two anionically stabilized bitumen emulsions with styrene-acrylic, styrene-butadiene and natural rubber emulsions as well as self crosslinking ethylene-vinyl acetate, styrene-acrylic and acrylic emulsions. Paints prepared with these binders were characterized by drying time, hardness, flexibility, adhesion and other property determinations. As with solvent based ones, excess flexibility and low hardness values had to be compensated with fillers and other additives. Most of the formulations obtained yielded significantly superior water based paints in comparison with commercially available solvent based bitumen paints.

\section{Experimental}

\subsection{Materials}

Bitumen emulsion "Bitüm Teknik İzolasyon San. D1ş Tic. Ltd. Şti." was a low viscosity emulsion (325 mPa.s, Tg: $3^{\circ} \mathrm{C}$ ) obtained from high penetration grade bitumen (E1). The second one was "Orcan A.Ş.; Orkote" prepared from higher penetration bitumen with bentonite additive and high viscosity (6560 mPa.s, Tg: $\left.-1^{\circ} \mathrm{C}\right)(\mathrm{E} 2)$. Styrene-acrylic emulsions were Betapol SA $5017 \mathrm{~B}$, (50\% solids, viscosity $7500 \mathrm{mPa} . \mathrm{s}$ Tg: $\left.17^{\circ} \mathrm{C}\right)(\mathrm{SA}-1)$ and Betapol SA $505(50 \%$ solids, viscosity $4500 \mathrm{mPa} . \mathrm{s}, \mathrm{Tg}:-3^{\circ} \mathrm{C}$ ) (SA-2). Styrenebutadiene rubber emulsion was Synthomer 20W20, (55\% solids, viscosity: $\left.450 \mathrm{mPa} . \mathrm{s}, \mathrm{Tg}:-35^{\circ} \mathrm{C}\right)(\mathrm{S} / \mathrm{B})$, natural rubber emulsion was "Kabuk Kauçuk" (60\% solids, viscosity: $800 \mathrm{mPa} . \mathrm{s}$, Tg: $-10.5^{\circ} \mathrm{C}$ ) (NR), crosslinking ethylene-vinyl acetate emulsion was Vinamul $3231 \quad(50 \%$ solids, viscosity: $\left.300 \mathrm{mPa} . \mathrm{s}, \quad \mathrm{Tg}:-7^{\circ} \mathrm{C}\right)$ (EVA-X), crosslinking styrene-acrylic emulsion was Acronal LR 9014 (45\% solids, viscosity: $\left.250 \mathrm{mPa} . \mathrm{s}, \mathrm{Tg}:-8^{\circ} \mathrm{C}\right)$ (SA-X), crosslinking acrylic emulsions were Betapol BPA 2520 (45\% solids, viscosity: $\left.500 \mathrm{mPa} . \mathrm{s}, \mathrm{Tg}: 29^{\circ} \mathrm{C}\right)(\mathrm{A}-\mathrm{X}-1)$ and Betapol BPA 2545 (45\% solids, viscosity: $400 \mathrm{mPa} . \mathrm{s}, \mathrm{Tg}$ : $44^{\circ} \mathrm{C}$ ) (A-X-2), Oxazoline functional polymer was Epocross WS500 (39\% solids, oxazoline value: $4.5 \mathrm{mmol} / \mathrm{g}$ nonvolatile, $\left.\mathrm{Tg}: 50^{\circ} \mathrm{C}\right)(\mathrm{OX})$.

The black pigments were iron oxide Bayferrox $31 \mathrm{M}$ (Size: $0.2 \mu \mathrm{m}$, s.g: 4.2 ) and carbon black Printex U (bulk density: 0.2, s.g: 1.8). The filler was Feldspar PR-C5 (Size: $5 \mu \mathrm{m}$, s.g: 2.6). Also various rheological and stabilizing additives were used. They are given below, in paint preparation section

\subsection{Bitumen Modifications}

Modifications were carried out by blending of bitumen and modifying emulsions in a high speed disperser at 1000 rpm for 30 minutes. They were filtered and used for paint preparations.

\subsection{Paint Preparations}

Paints were prepared in $2 \mathrm{~L}$ high speed dispersers followed by pearl mill grinding, to below $25 \mu \mathrm{m}$ size. It was carried out in conventional paint preparation procedure, where pigments and fillers were dispersed initially with appropriate amounts of dispersing aids, followed by addition of binders and other additives.

Base formulation was $50 \%$ modified bitumen emulsion (50\% solids), 5.6\% Bayferrox 316, 0.2\% Printex U, 30\% Feldspar PR-C5, 0.15\% each Calgon N and Coatex P 90 (wetting agents), $0.25 \%$ BYK 034 (defoamer), 1.5\% Bentone EW Paste (thickener), 0.2\% Acticide BX (N) (biocide), $0.1 \%$ Metolat 285 (substrate wetting agent), $0.1 \%$ Ascotran H-10 (flash rust inhibitor), $11.85 \%$ water. This water based formulation gave flat black paints with a PVC of $34 \%$.

\subsection{Tests and Measurements}

The paints were applied on steel panels and the physical properties were determined after 1 week. Hardness was determined by König pendulum (TS6041/EN ISO 1522). Adhesion was determined by cross-cut test (TS4313/EN ISO 2409). Flexibility was determined by mandrel bending test (TS39/TS 8693). Gloss was determined according to TS 4318/EN ISO 2813. Dry to touch times were determined according to ASTM 1640. For the dry hard time, the more strict method of "coin mar test" was found to be more appropriate, since the main use bitumen paints was on pipes, which involved stacking on top each other as soon as possible. This was a "pass or fail" test, and it was found that when a pass was obtained, the coating had already reached the 7th degree of drying of DIN 53150. The light microscopy images were obtained from $60 \mu \mathrm{m}$ dry films of modified bitumens on glass at 50x magnification by Nikon Eclipse LV 100. Image analysis was realized by Vision Lite Software. Fluorescence Microscopy images were obtained by Olympus BX-51 TRF-5. Tg measurement was carried out with $10 \mathrm{mg}$ samples at a heating and cooling rate of $10^{\circ} \mathrm{C} / \mathrm{min}$ by Perkin Elmer DSC 6000 , under nitrogen flow. The images given are those of blends of unpigmented samples. Viscosity was measured by Brookfield DVE viscometer at $20^{\circ} \mathrm{C}$ with spindle $2-7$ at $20 \mathrm{rpm}$. L, a, b color parameters were determined by X-rite Color i5 Benchtop Spectrophotometer.

\section{Results and Discussion}

Our previous research [4] has shown that modification of bitumen physically by adding polymers or reacting with prepolymers containing glycidyl or isocyanate groups significantly improves the properties of solvent based paints prepared by using them as the main binder. Compared to modification of bitumens for these solvent systems, which involved heating under toluene reflux for several hours, preparing the emulsion blends under ambient conditions with high shear mixers was relatively easy. On the other hand, preparation of the paints from these blends was somewhat harder, requiring careful choice of fillers, flash rust and stabilizing additives. Reaching a satisfactory 
grinding stage and avoiding high viscosities were possible with feldspar as the main filler, rather than baryte or calcite.

But once a satisfactory formulation was available, some of the results were better than expected. Modification by blending with styrene-acrylic, styrene-butadiene or natural rubber emulsions, as seen from Table $1 \mathrm{a}$ and $1 \mathrm{~b}$, gave paints with excellent dry to touch and dry hard times, often accompanied by good adhesion and flexibility properties. All percentages are given on weight basis. Both bitumen and modifying emulsions had similar surfactants at similar concentrations therefore their effect can be ignored.

Table 1a. Properties of paints prepared from physical modification of E1 bitumen emulsions

\begin{tabular}{|c|c|c|c|c|c|}
\hline Experiment & 1 & 2 & 3 & 4 & 5 \\
\hline Bitumen Emulsion & E-1 & E-1 & E-1 & E-1 & E-1 \\
\hline Modifier & S/A-1 & S/A-1 & $\mathrm{S} / \mathrm{A}-2$ & $\mathrm{~S} / \mathrm{B}$ & NR \\
\hline Modification \% & 10 & 20 & 10 & 10 & 10 \\
\hline Dry to Touch (min) & 30 & 120 & 35 & 25 & 20 \\
\hline Dry Hard (h) & 3,5 & 6 & 4 & 3,5 & 3,5 \\
\hline Adhesion* & 1 & 2 & 0 & 1 & 1 \\
\hline Hardness (sec) & 9 & 16 & 6 & 7 & 7 \\
\hline Gloss $\left(60^{\circ}\right)$ & 1 & 1 & 1 & 1 & 1 \\
\hline Flexibility** & 0 & 0 & 0 & 0 & 0 \\
\hline
\end{tabular}

* 0: Best, 5: Worst; ** 0: Best, 6: Worst

Table 1b. Properties of paints prepared from physical modification of E2 bitumen emulsions

\begin{tabular}{|c|c|c|c|c|c|c|c|}
\hline Experiment & 6 & 7 & 8 & 9 & 10 & 11 & 12 \\
\hline Bitumen Emulsion & E-2 & E-2 & E-2 & $\mathrm{E}-2$ & $\mathrm{E}-2$ & E-2 & E-2 \\
\hline Modifier & S/A-1 & S/A-1 & S/A-1 & S/A-1 & $\mathrm{S} / \mathrm{A}-2$ & $\mathrm{~S} / \mathrm{B}$ & NR \\
\hline Modification \% & 10 & 15 & 20 & 30 & 10 & 10 & 10 \\
\hline Dry to Touch (Min) & 25 & 80 & 90 & 60 & 30 & 40 & 30 \\
\hline Dry Hard (H) & 2 & 3 & 3,5 & 3 & 3 & 3 & 2 \\
\hline Adhesion* & 0 & 2 & 1 & 0 & 0 & 0 & 2 \\
\hline Hardness (sec) & 24 & 28 & 40 & 42 & 13 & 11 & 14 \\
\hline Gloss $\left(60^{\circ}\right)$ & 1 & 1 & 1 & 1 & 1 & 1 & 1 \\
\hline Flexibility** & 0 & 0 & 0 & 0 & 0 & 0 & 0 \\
\hline
\end{tabular}

*0: Best, 5: Worst; ** 0: Best, 6: Worst

Since the modifiers had low $\mathrm{T}_{\mathrm{g}}$ values, film formation was easy, but they yielded soft films, with the exception of $\mathrm{S} / \mathrm{A}-1$, which had a $\mathrm{T}_{\mathrm{g}}$ of $17^{\circ} \mathrm{C}$. In this case, by increasing the amount of S/A-1 in E-2 blends, it was possible to attain a König hardness of 42 seconds (Exp. 9). The change in morphology can be observed from fluorescence spectroscopy images given in Fig. 1. The light regions represent the polymer rich phase and the darker regions the bitumen rich phase. Some groups of the bitumen also give a slight fluorescence (Fig. 1a). For 10\% S/A-1, one observes a homogeneous distribution of the polymer in the bitumen matrix (Fig. 1b). At 20\%, the polymer rich phase, absorbing maltene from bitumen, swells and the system becomes heterogeneous (Fig. 1c). At the highest concentration of $30 \%$, the polymer rich phase is the dominant matrix and larger size asphaltene rich bitumen phase regions can be observed (Fig. 1d).
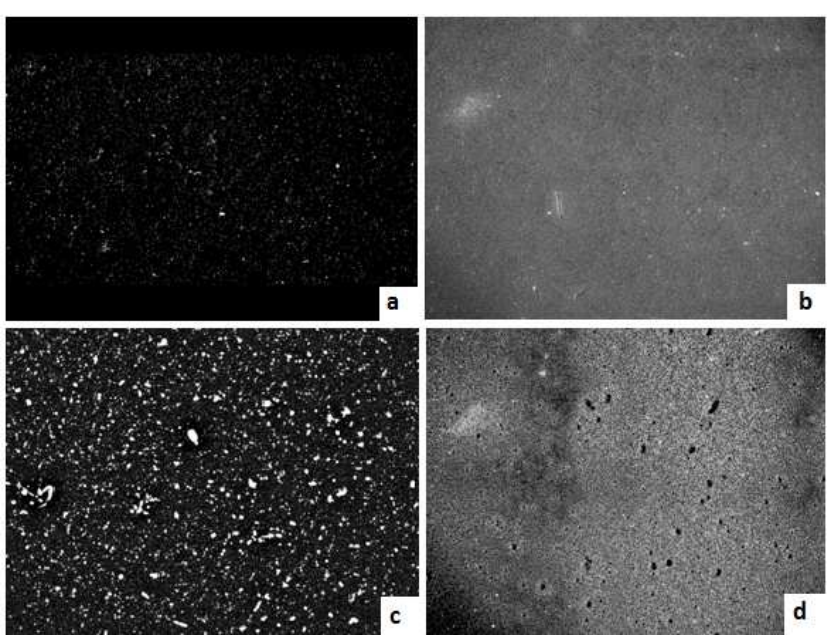

Figure 1. Fluorescence microscopy results of blends of E2 (a), with $S / A-1$ (b) $10 \%$, (c) $20 \%$, (d) $30 \%$ 

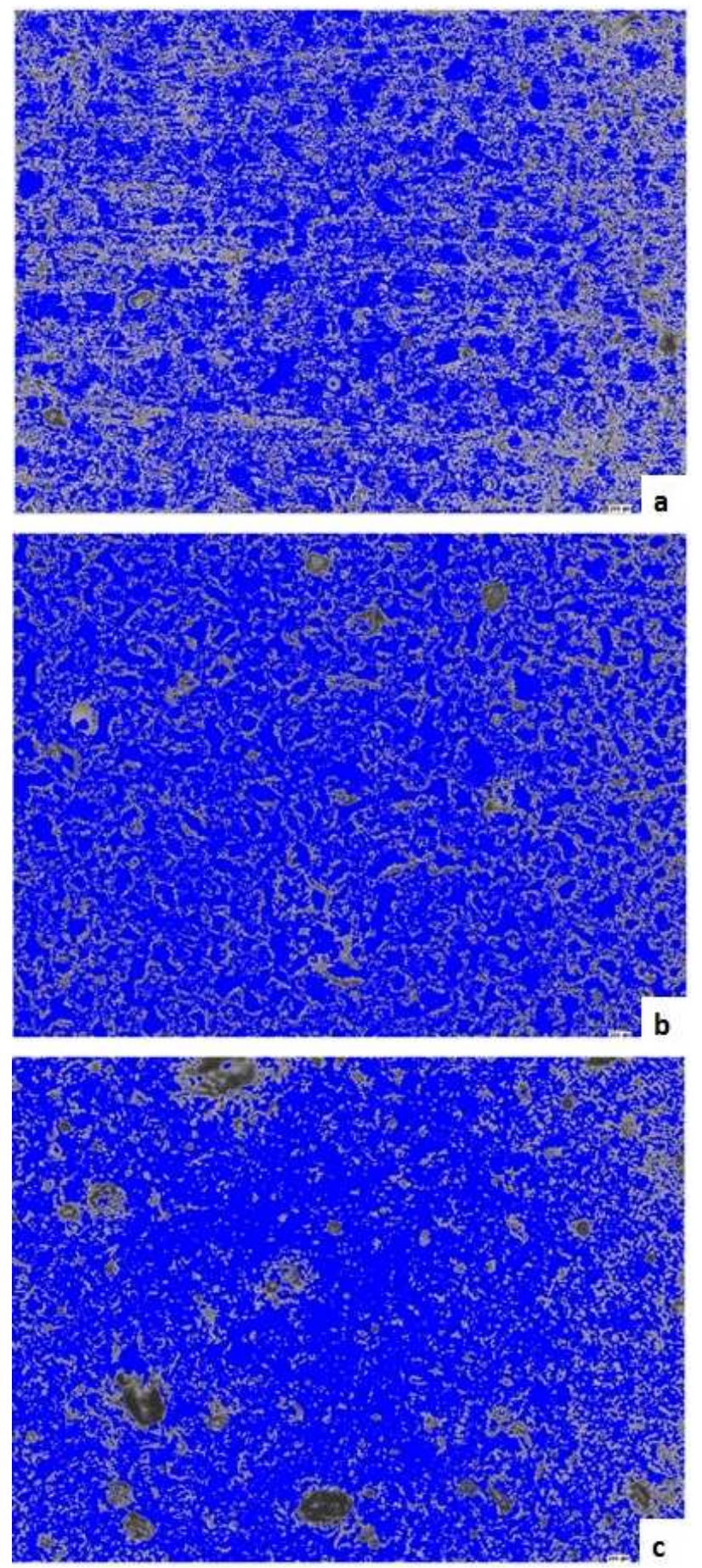

Figure 2. Light microscopy-image analysis(50x) results of blends of E2 with $S / A-1$, (a) $10 \%$, (b) $20 \%$, (c) $30 \%$

The chemical modification attempts of bitumen emulsions by crosslinking EVA, styrene-acrylic and acrylic emulsions did not provide better results than physical ones, contrary to the expectations (Table 2a and 2b). E-1 emulsion blends were again softer than E-2 ones, A-X-1 with the highest $\mathrm{T}_{\mathrm{g}}$ of $29^{\circ} \mathrm{C}$ providing 16 and $40 \mathrm{König}$ seconds hardness values for E-1 and E-2, respectively (Exp. 16 and 22). Obviously the degree of crosslinking was low or nonexistent and in some cases (Exp. 21 and 22) serious adhesion loss was observed. Addition of oxazoline to S/A-1 modified E-2 (Exp. 24 and 25) did provide some crosslinking, as the hardness values slightly increased, but unfortunately along with a complete loss of adhesion, even at $1 \%$ oxazoline addition.

The microscopy images given in Fig. 3 show the morphological changes by blending bitumen emulsions with crosslinking (A-X-1) or non crosslinking (S/A-1) emulsions. In case of E-2, bentonite stabilization apparently forced asphaltene rich darker regions to separate, similar to polymer additions to bitumens [4]. When E-1 was modified with S/A-1, the polymer rich phase absorbing the MAL fraction brought a similar appearance of ASP rich phase, but to a lesser degree. With A-X-1, a similar picture indicated a modification like S/A-1 rather than crosslinking, but some additional flakes did appear. When E-2 was blended with S/A-1ASP rich phase was distributed better, indicating good compatibility. On the other hand, modification with A-X-1 gave a picture very similar to unmodified E-2.

Normal microscopy combined with image analysis, which calculates the areas of darker and lighter colored regions, confirms this change in morphology (Fig. 2), where the darker colored area increases up to $75 \%$, in case of $30 \% \mathrm{~S} / \mathrm{A}-1$.
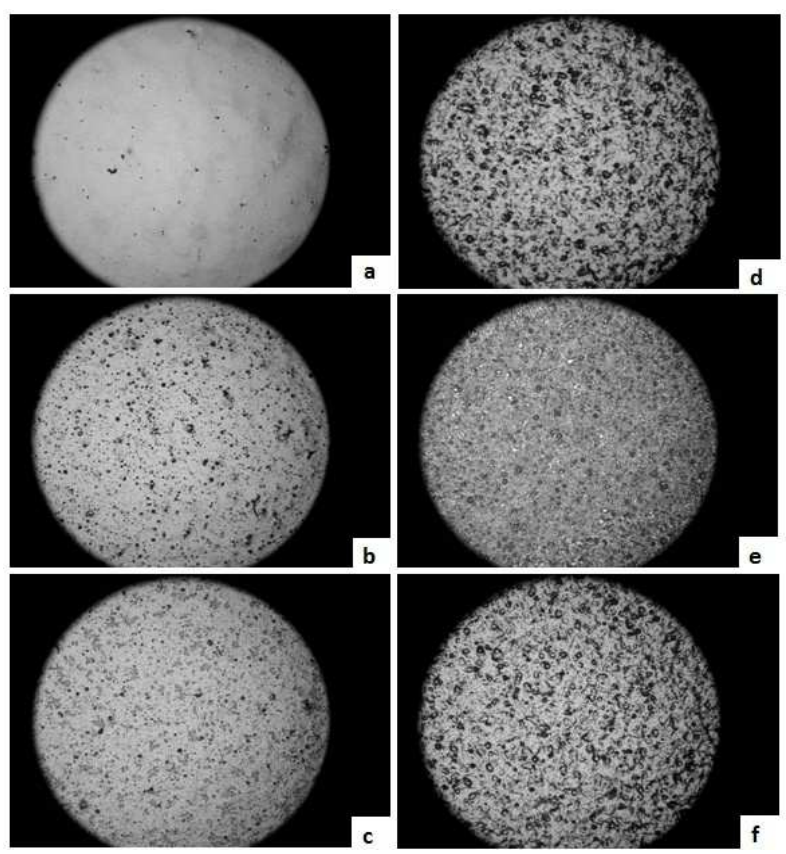

Figure 3. Light Microscopy images of (a) E-1, (b) E-1 blend with S/A-1, (c) E-1 blend with $A-X-1$, (d) E-2, (e) E-2 blend with $S / A-1,(f) E-2$ blend with $A-X-1$ 
Table 2a. Properties of paints prepared from chemical modification of E1bitumen emulsions

\begin{tabular}{|c|c|c|c|c|c|}
\hline Experiment & 13 & 14 & 15 & 16 & 17 \\
\hline Bitumen Emulsion & E-1 & E-1 & E-1 & E-1 & E-1 \\
\hline Modification Agent & EVA-X & S/A-X & A-X-1 & A-X-1 & A-X-2 \\
\hline Modification \% & 10 & 10 & 10 & 20 & 10 \\
\hline Dry to Touch (min) & 30 & 30 & 90 & 100 & 90 \\
\hline Dry Hard (h) & 2.5 & 9 & 2 & 4.5 & 2.5 \\
\hline Adhesion* & 0 & 0 & 0 & 1 & 2 \\
\hline Hardness (sec) & 7 & 8 & 11 & 16 & 9 \\
\hline $\operatorname{Gloss}\left(60^{\circ}\right)$ & 1 & 1 & 2 & 2 & 1 \\
\hline Flexibility** & 0 & 0 & 0 & 0 & 0 \\
\hline
\end{tabular}

* 0: Best, 5: Worst; ** 0: Best, 6: Worst

Table $2 \boldsymbol{b}$. Properties of paints prepared from chemical modification of E2bitumen emulsions

\begin{tabular}{|c|c|c|c|c|c|c|c|c|}
\hline Experiment & 18 & 19 & 20 & 21 & 22 & 23 & 24 & 25 \\
\hline Bitumen Emulsion & E-2 & E-2 & E-2 & E-2 & E-2 & E-2 & E-2 & E-2 \\
\hline Modification Agent & EVA-X & S/A-X & A-X-1 & A-X-1 & A-X-1 & A-X-2 & $\mathrm{S} / \mathrm{A}-1+\mathrm{OX}$ & $\mathrm{S} / \mathrm{A}-1+\mathrm{OX}$ \\
\hline Modification \% & 10 & 10 & 10 & 15 & 20 & 10 & $10+1$ & $10+4$ \\
\hline Dry to Touch (min) & 30 & 30 & 35 & 60 & 90 & 90 & 90 & 120 \\
\hline Dry Hard (h) & 1.5 & 6 & 6.5 & 3 & 3.5 & 2 & 3 & 3 \\
\hline Adhesion* & 0 & 0 & 0 & 3 & 4 & 2 & 5 & 5 \\
\hline Hardness (sec) & 15 & 24 & 25 & 26 & 40 & 9 & 33 & 35 \\
\hline Gloss $\left(60^{\circ}\right)$ & 1 & 1 & 1 & 1 & 1 & 1 & 1 & 1 \\
\hline Flexibility** & 0 & 0 & 0 & 0 & 0 & 0 & 0 & 0 \\
\hline
\end{tabular}

* 0: Best, 5: Worst; ** 0: Best, 6: Worst

Bitumen paints are notorious for their unfortunate bleeding properties. It is almost impossible to apply a solvent based top coat on bitumen based primer paint without disrupting the primer, due to the solubility of bitumen fractions in common paint solvents. Consequently, when a top layer is applied over dry bitumen paint, the MAL fraction diffuses to this layer, causing extensive discoloration. Thus we would also like to report an encouraging result of a preliminary experiment, where a water based white alkyd/acrylic blend paint was applied over films of $20 \%$ and $30 \%$ S/A-1 containing paints ( Exp. 8 and 9). The CIE "L", "a", "b" parameters for (I) double applications of 60 $\mu \mathrm{m}$ dry films of water based white paint and same thickness of (II) Exp. 8 and (III) Exp. 9 paints, plus a top coat of the same white paint were found to be (I) $\mathrm{L}=91.47, \mathrm{a}=-1.22$, $\mathrm{b}=4.40$; (II) $\mathrm{L}=89.39, \mathrm{a}=-1.21, \mathrm{~b}=4.40$; (III) $\mathrm{L}=89.39$, $\mathrm{a}=$ $-1.24, \quad b=4.32$, respectively, after 3 months at under ambient conditions. These show small $\Delta \mathrm{E}$ differences of 2.08 and 1.70 for Exp. 8 and 9, respectively, due to change in lightness parameter L, which in turn was mainly caused by the black color of the primer layers. On the other hand, the "a", the redness-greenness and "b", yellownessblueness parameters differences are negligible, confirming that no bleeding of the dark colored MAL occurred.

\section{Conclusion}

Bitumen based solvent paints still survive due to their excellent adhesion and corrosion protection properties as well their incomparably cheap price, as a binder for large area applications, such as pipelines. But available unsophisticated paints prepared from bitumen-filler-resin combinations show deficiencies in either in drying time and hardness or adhesion and flexibility. On the other hand, a previous report on a scientific investigation of a water based version is not in existence. Our previous work has shown that improving solvent based paint properties by physical or chemical modification by polymers was possible. Applying this approach for water based paints has shown that advance in properties such as hardness and drying times without loss in adhesion and flexibility can be obtained by blending with various polymeric emulsions with bitumen emulsions, normally used for waterproofing membranes. Physical modifications with styrene acrylic plastomers and styrene butadiene and natural rubber elastomers were carried out with two different bitumen emulsions. Changes in morphology of the films occurred after blending, which can be seen from fluorescence and normal microscopy, indicating interaction between bitumen 
maltene fractions and polymers. These bitumen emulsions in unmodified form gave very soft paint films which did not dry. On the other hand, especially after modification with styrene acrylic emulsions some very satisfying results in hardness and drying time without loss in adhesion and flexibility was obtained. Modifications with crosslinking emulsions also provided some good paints but impairing of adhesion was a problem.

An application of water based alkyd/acrylic blend paint over two modified paints proved that the bleeding problem of these paints can be avoided, for the first time. Thus further research on employment of these as primers for other topcoats seems worthwhile.

\section{Acknowledgement}

This work was supported by TÜBİTAK-TEYDEB under project number 3090648. Authors are grateful to TEYDEB for financial support as well as Prof. Dr. İsmail Boz and İSFALT A.Ş. laboratories, İstanbul, Turkey for fluorescent microscopy.

\section{References}

[1] D. Lesueur, Adv. Colloid Interface Sci. 145, (2009) 42-82.

[2] Y. Yildirim, Construct. Build. Mater. 21, (2007) 66-72.

[3] A.P. Kuriakose, S. Kochu Baby Manjooran, Surf. Coat. Technol.145, (2001) 132-138.

[4] R. Aydemir, M. Eren, H. Aşkun, A. E. Özbey, M. Orbay, Prog. Org. Coat. 76, (2013) 966-971 